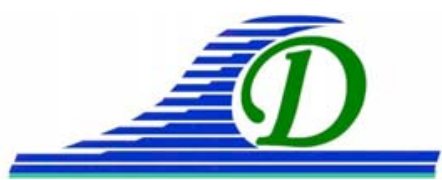

XIII ${ }^{\text {èmes }}$ Journées Nationales Génie Côtier - Génie Civil Dunkerque, 2-4 juillet 2014

DOI:10.5150/jngcgc.2014.026 (C) Editions Paralia CFL

disponible en ligne - http://www.paralia.fr - available online

\title{
Evolution mi-séculaire des côtes sédimentaires du département du Finistère
}

\section{Emmanuel BLAISE ${ }^{1}$, Serge SUANEZ ${ }^{1}$, Jean-Michel SCHROETTER ${ }^{2}$, Eric PALVADEAU ${ }^{2}$}

\section{GEOMER - LETG UMR 6554 CNRS - IUEM, Technopôle Brest Iroise, Place}

Nicolas Copernic, 29280 Plouzané, France.

emmanuel.blaise@univ-brest.fr ; serge.suanez@univ-brest.fr,

2. BRGM Bretagne, 2 rue de Jouanet, 35700 Rennes, France.

jm.schroetter@brgm.fr ; e.palvadeau@brgm.fr

\section{Résumé}

Cette étude a été réalisée dans le cadre d'un projet de constitution d'un "Atlas des aléas littoraux pour les départements bretons", commandité par la Région Bretagne. Elle porte sur l'aléa érosion du trait de côte, et présente une analyse diachronique de l'évolution de la ligne de rivage sur soixante ans (1952-2009), pour l'ensemble des côtes sédimentaires (sableuses et de galets) du littoral du Finistère en Bretagne (hors îlots non habités). D’un point de vue méthodologique, un inventaire basé sur des indicateurs morphosédimentaires nous a permis dans un premier temps d'établir une typologie des côtes sédimentaires; par la suite, nous nous sommes basés sur les principes de photointerprétation pour évaluer l'évolution du trait de côte. Les variations observées sur la période coïncident avec les résultats obtenus par l'Ifen (Institut français de l'environnement) en 2003 portant sur l'ensemble du trait de côte français. L'érosion agissant différemment suivant le type de côte, nous avons montré que les plages ouvertes constituées de cordons dunaires étaient beaucoup plus affectées que les cordons sableux adossés, situés en position d’abri au fond des baies.

Mots-clés : Aléa érosion, Côtes sédimentaires, Trait de côte, Photo-interprétation, Finistère.

\section{Introduction}

Cette étude s'inscrit dans un projet d'élaboration d'un atlas des aléas littoraux pour les départements de l'Ille-et-Vilaine, des Côtes d'Armor et du Finistère. Ce projet, commandité par la DREAL Bretagne, s’inscrit dans le contexte de gestion intégrée de la zone côtière (GIZC) et a pour objectif principal d'améliorer (i) la caractérisation morpho-sédimentaire des types de côte, (ii) la connaissance des aléas littoraux en matière d'érosion et de submersion marine. Elle a été réalisée en collaboration avec l'antenne du BRGM de Rennes; l'objectif étant de fournir une cartographie de l'évolution du trait de côte des littoraux meubles à l'échelle des soixante dernières années pour les trois départements bretons. 


\section{Thème 2 - Dynamique sédimentaire}

En effet, les seules données actuellement disponibles sur la mobilité du trait de côte pour l'ensemble de la Bretagne consistent en une cartographie réalisée sur la période 1998-2003 dans le cadre d'un programme régional appelé EROCOVUL (HENAFF et al., 2006). Bien qu'utile, cette information reste toutefois peu représentative des dynamiques à long terme lorsque l'on sait que l'action des épisodes tempétueux à très court terme intervient de façon prépondérante dans l'évolution des littoraux meubles. Or, la période considérée dans cette étude inclue les fortes tempêtes de la fin de la décennie 1990 et du début des années 2000 (HENAFF et al., 2006). Pour autant, les données produites dans le cadre de ce travail ont montré que sur 30\% du linéaire renseigné (soit $1283 \mathrm{~km}$ sur $4324 \mathrm{~km}$ incluant les îles, les ilots et estuaires), 22\% du littoral était en érosion, $72 \%$ stable, et seulement $6 \%$ en accrétion. A cela, s'ajoute un nombre important d'études ponctuelles réalisées sur des secteurs le plus souvent très restreints et dont il serait vain d'en faire l'énumération. Mais là encore, il est difficile d'en faire une synthèse globale car ces travaux couvrent au final une portion de linéaire côtier très réduite. De même, les méthodes d'analyse et/ou les indicateurs morphologiques du trait de côte utilisés dans ces études peuvent varier d'un auteur à l'autre, ce qui rend très difficile les comparaisons d'un site à l'autre. Il apparaissait donc nécessaire de reprendre ce travail d'analyse de l'évolution du trait de côte en adoptant un protocole méthodologique homogène à l'ensemble des sites étudiés, et en étendant la période d'observation.

L'étude que nous avons effectuée porte sur la quantification de l'évolution du trait de côte des cordons meubles à l'échelle des soixante dernières années. Pour ce faire, deux missions de photographies aériennes ortho-rectifiées de 1952 et de 2009 ont été utilisées. La première partie du travail a consisté à établir une classification des différents types de côtes sédimentaires en fonction de la morphologie et de la nature du matériel de ces dernières. Dans un second temps, il s'est agi de définir un (ou des) indicateur(s) permettant de définir la limite du trait de côte en fonction des caractéristiques morpho-sédimentaires du milieu. Enfin, le travail de numérisation et de quantification de la cinématique du trait de côte a été réalisé sous ArcGIS en suivant un protocole méthodologique aujourd'hui bien connu (DOLAN et al., 1978; CROWELL et al., 1991 \& 1997 ; SHOSHANY et al., 1992 ; FENSTER et al., 1993 ; DOUGLAS et al., 1998 ; MOORE, 2000). Dans le cadre de cet article, nous ne présenterons que les résultats obtenus pour le département du Finistère.

\section{Site d'étude}

Le département du Finistère est situé à l'extrémité ouest de la Bretagne. Il est bordé au nord par la Manche, à l'ouest par la mer d'Iroise et au sud par l'Océan Atlantique (figure 1). Son linéaire s'étend de la baie de Lannion au nord, à l'estuaire de la Laïta au sud, sur près de $1380 \mathrm{~km}$ faisant de lui le département français possédant le plus long littoral (HALLEGOUËT et al., 1986). 


\section{XIII ${ }^{\text {èmes }}$ Journées Nationales Génie Côtier - Génie Civil \\ Dunkerque, 2-4 juillet 2014}

La morphologie générale s’organise autour des trois façades littorales que sont :

- la côte nord qui s'étend de la pointe de Corsen à la baie de Lannion, marquée par l'alternance de côtes sableuses et rocheuses dessinant un linéaire extrêmement découpé. Cette irrégularité s’apparente aux processus de "contraposition", elle-même à l'origine de la mise en place de grandes formes d'accumulation constituées de cordons sableux et de galets (GUILCHER, 1948). On y trouve également les grands abers constitués principalement des rivières du Dossen et de la Penzé (baie de Morlaix), ou plus à l'ouest, des abers Wrac'h, Benoit et de Lanildut.

- la côte ouest se distingue par la présence de hautes falaises notamment en presqu'île de Crozon où elles culminent à presque $100 \mathrm{~m}$ d'altitude (Cap de la chèvre). Les formes d'accumulation sont quant à elles cantonnées aux larges rentrants que dessine cette côte à l'exemple de la rade de Brest et ses nombreux petits estuaires, la baie de Douarnenez ou d'Audierne.

- le sud Finistère est quant à lui essentiellement constitué de grands cordons sableux dunaires fermant de vastes zones basses situées en arrière, à l'image des cordons de Penmarc'h, Treffiagat, Combrit, Mousterlin et Tregunc. On y trouve aussi de grandes rias très évasées prenant l'allure de baie comme celle de Bénodet creusée par les rivières de Pont-l'Abbé et l'Odet.

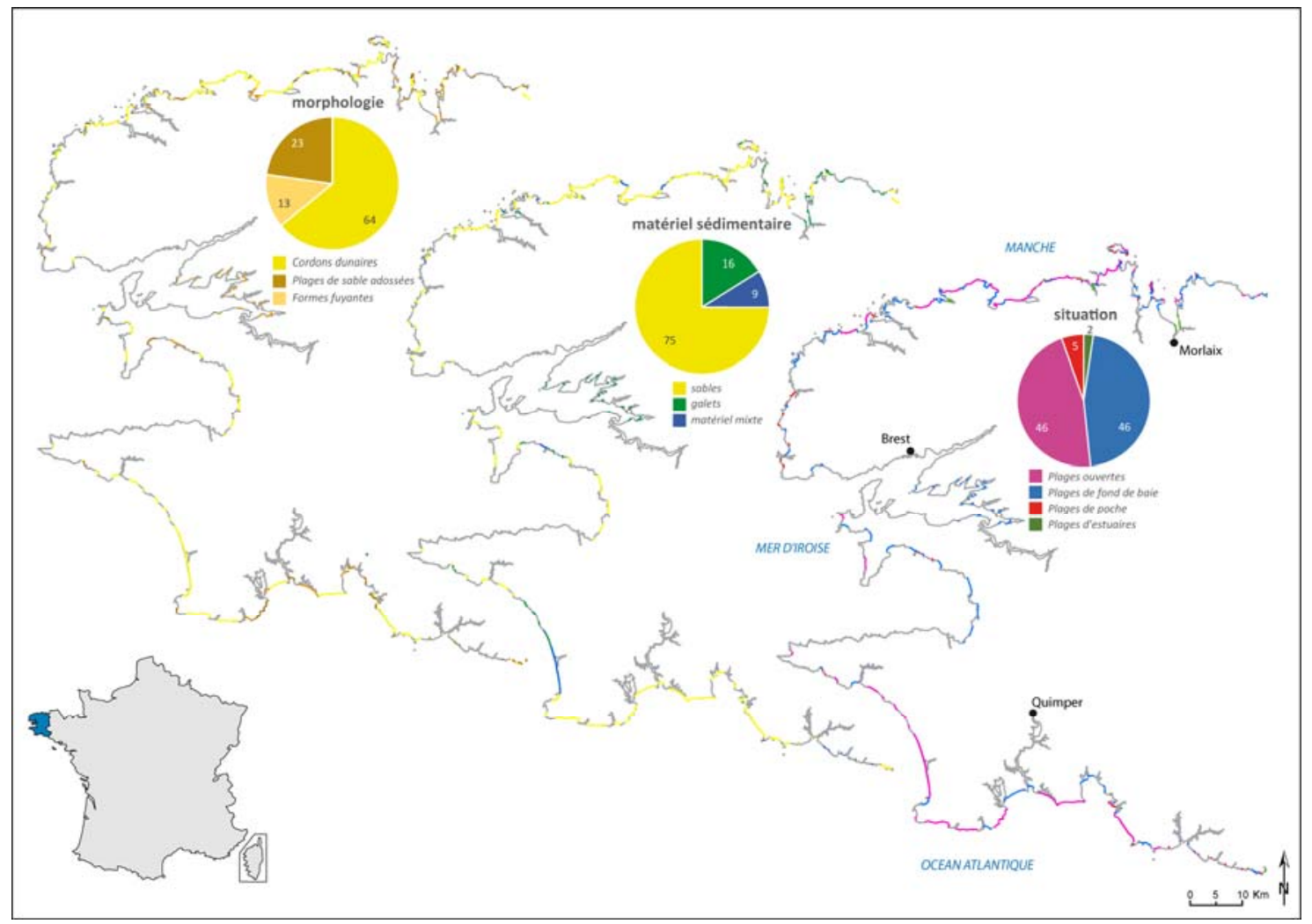

Figure 1. Physionomie des côtes sédimentaires du Finistère. 


\section{Thème 2 - Dynamique sédimentaire}

\section{Inventaire des différents types de côte sédimentaires}

Cet inventaire repose sur un certain nombre d'éléments morpho-sédimentaires. Nous avons distingué (i) la "situation" illustrant l'exposition du cordon littoral en tant que plages de fond de baie, plages ouvertes, d'estuaire ou de poche. Le critère sédimentologique en tant que (ii) "matériel" a constitué le deuxième paramètre permettant de distinguer les sables des galets ainsi que le matériel mixte. Le troisième élément a concerné (iii) "la morphologie" différenciant les plages adossées à la falaise, des cordons dunaires, des formes fuyantes (flèches de sables et de galets, tombolos). Le degré d'anthropisation du trait de côte (iv) a finalement été pris en compte afin de déterminer l'artificialisation de ces milieux.

En raison d'une méthodologie de cartographie et de comparaison propre aux marais maritimes, ces derniers n’ont pas été pris en compte dans l'étude.

\section{Choix d'une ligne de référence}

En géomorphologie, le trait de côte repose obligatoirement sur une limite physiographique "fixe" que l’on peut suivre dans le temps et dans l'espace. Néanmoins, cette limite diffère en fonction du type de côte et du contexte marégraphique. De très nombreuses études portant notamment sur l'analyse de la cinématique du trait de côte ont proposé un très large éventail de ligne de référence pouvant être classées en trois grandes familles que sont les indicateurs morphologiques, biologiques, et la position des différents niveaux d'eau (BOAK \& TURNER, 2005).

Un des principaux objectifs de cette étude était de définir une limite du trait de côte qui tienne compte des différents types d'environnements littoraux identifiés dans la typologie présentée précédemment (figure 2). Ainsi, la limite de végétation dunaire a été retenue pour les cordons dunaires. Dans ce cas, elle peut aussi correspondre (i) au somment de l'escarpement de falaise lorsque la dune est érodée, (ii) à la limite des dunes embryonnaires de haut de plage lorsque le système plage/dune est en accrétion (figure 2).

La limite de végétation constitue ici l'indicateur le plus pertinent puisqu'il s'agit d'étudier les dynamiques du trait de côte en milieux dunaires en milieu macrotidal. Ceci s'explique principalement par la présence d'un contraste suffisamment élevé et aisément perceptible entre les pixels clairs et obscurs des deux types de photographies. La frontière entre le sable et la végétation dunaire est ainsi facilement identifiable (MOORE et al., 1999 ; PRIEST, 1999 ; BOAK \& TURNER, 2005). Par ailleurs, la végétation saisonnière quasi invisible sur les photographies aériennes anciennes, est facilement dissociable de la végétation pérenne sur les ortho-photographies de 2009.

La végétation supralittorale a été choisie pour les cordons de galets "végétalisés" ainsi que pour les crêtes sommitales falaises meubles. Pour les falaises rocheuses, le pied de falaise ou le haut de falaise (suivant le contraste et l'exposition de l'ortho-photographie de 1952) ont été retenues. Pour les formes fuyantes mobiles telles que les flèches de 


\section{XIII ${ }^{\text {èmes }}$ Journées Nationales Génie Côtier - Génie Civil \\ Dunkerque, 2-4 juillet 2014}

galets à pointe libre, c'est le pied du revers qui a été choisi (STEPHAN, 2011). Pour finir, lorsque le trait de côte était fortement anthropisé, le contact entre la plage et l’ouvrage a été retenu (MOORE et al., 1999).
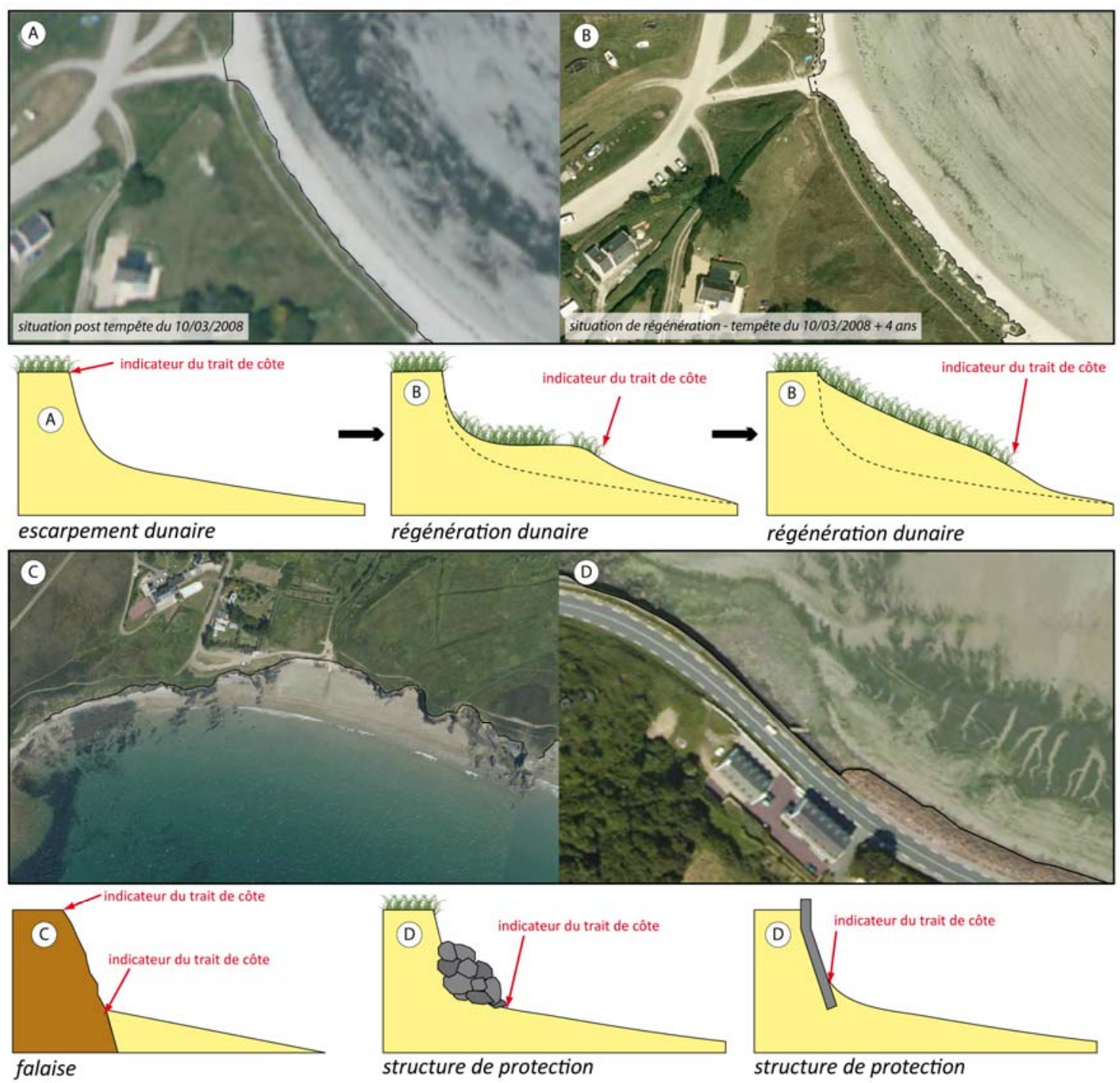

Figure 2. Indicateurs du trait de côte utilisés dans cette étude.

\section{Evolution du trait de côte par photo-interprétation}

La digitalisation du trait de côte a été réalisée à même l'écran à l'aide du logiciel de SIG ArcGIS, à partir de deux séries d'ortho-photographies IGN (missions de mai à juin 1952 et de mai 2009) référencées en Lambert93. Les marges d'erreur inhérentes à l'orthocorrection et au géo-référencement des photographies aériennes, ainsi qu'à la digitalisation du trait de côte, ont été estimées à +/-5 m.

L'analyse quantitative du trait de côte a été réalisée à l'aide de l'extension ArcGIS, Digital Shoreline Analysis System (DSAS), développé par l'USGS (DOUGLAS et al., 


\section{Thème 2 - Dynamique sédimentaire}

1998 ; MOORE, 2000 ; THIELER et al., 2009). Le principe de cette extension est de mesurer les évolutions entre les différentes positions des traits de côte grâce à une série de transects orthogonaux à la plage, disposés le long d'une "ligne de base".

\section{Résultats}

\subsection{Physionomie des côtes finistériennes}

Les résultats montrent que les côtes sédimentaires du Finistère sont majoritairement composées de plages sableuses (75\%) (figure 1). Ces côtes sableuses sont à 64\% constituées de dunes comme le montrent les grands massifs dunaires du sud du Finistère (Pays Bigouden) et du nord du Léon. A cela s'ajoutent les cordons de plus petite taille en position d'abri, notamment sur la presqu'île de Crozon ou en baie de Douarnenez. On trouve enfin quelques formes fuyantes telles que les flèches dunaires de Penn ar C’hleuz (nord Finsitère) ou de Mousterlin (sud Finistère).

Les cordons de galets représentent quant à eux $16 \%$ des côtes meubles. Ils sont principalement situés en Rade de Brest ou en baie d'Audierne (cordon d'Ero Vili), ainsi que dans le Trégor finistérien (région de Morlaix). Les 9\% restant correspondent à des cordons littoraux dont la granulométrie est mixte (sables et galets).

\subsection{Evolution de la dynamique du trait de côte du Finistère de 1952 à 2009}

Les résultats montrent que 40\% du littoral finistérien (soit $90 \mathrm{~km}$ de côtes sur $220 \mathrm{~km}$ observés) sont en érosion ; 12\%, soit 27,5 km de linéaire côtier, sont stables ; 23\% du littoral (soit 20,5 km de linéaire) progradent. A cela s'ajoutent 23\% de linéaire côtier totalement artificialisé (figures 3 et 4). Pour ces derniers, il s'agit principalement d'aménagements de protection du littoral (enrochements, murets, etc.), mais également d'infrastructures portuaires telles que les quais et cales de mise à l'eau.

Dans le détail, la mobilité du trait de côte à l'échelle des 60 dernières années montre que le littoral évolue différemment selon le type de côtes. Bien évidemment, $45 \%$ des côtes sableuses formées d'un cordon dunaire (soit $64 \mathrm{~km}$ de linéaire côtier) reculent à la différence des plages adossées moins sensibles à l'érosion, dont le recul concerne seulement $28 \%$ de ce type de côte (soit $14 \mathrm{~km}$ de linéaire). On remarque également que $38 \%$ des formes fuyantes présentes sur le littoral reculent, soit $11 \mathrm{~km}$ du linéaire côtier étudié.

En comparant l'évolution du trait de côte des trois façades maritimes du Finistère, on observe une érosion plus forte sur la façade ouest (52\%) contre 35\% et 33\% respectivement pour les façades nord et sud. On remarque également que la façade ouest est la moins artificialisée (14\%). 


\section{XIII ${ }^{\text {èmes }}$ Journées Nationales Génie Côtier - Génie Civil \\ Dunkerque, 2-4 juillet 2014}

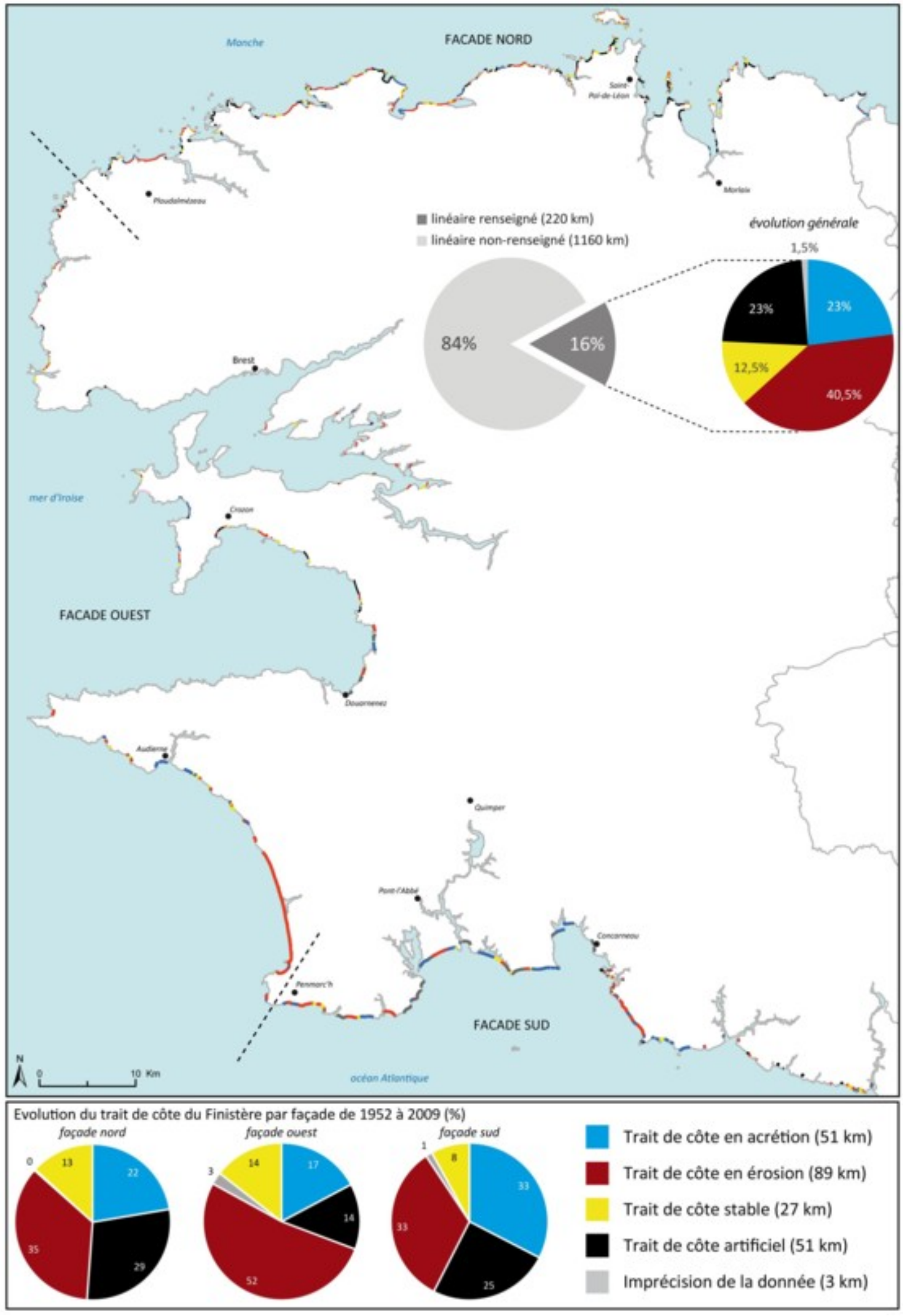

Figure 3. Evolution du trait de côte du Finistère de 1952 à 2009. 


\section{Thème 2 - Dynamique sédimentaire}

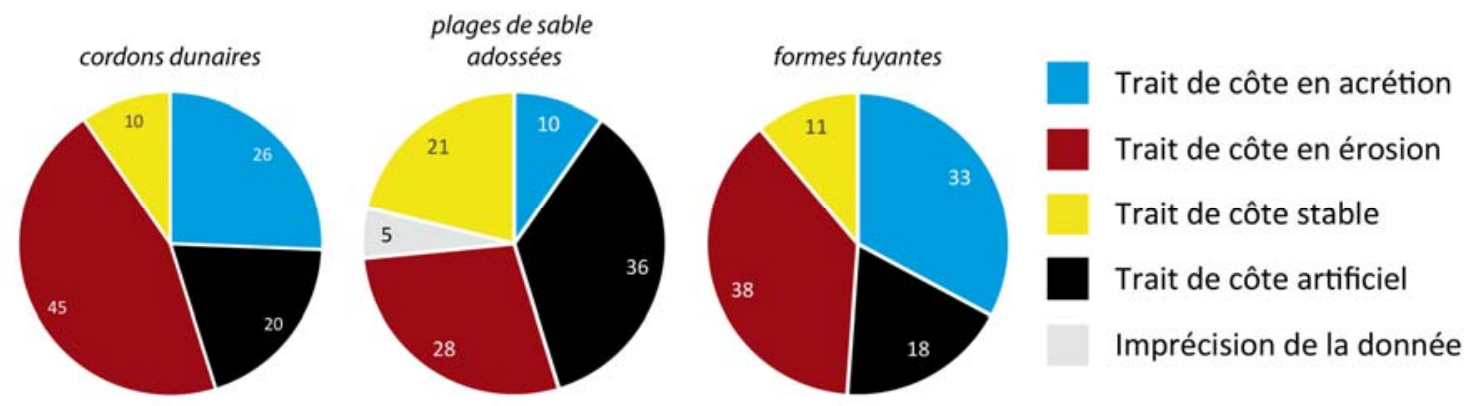

Figure 4. Evolution du trait de côte du Finistère de 1952 à 2009 en fonction du type de plage

\section{Discussion}

En 1985, Bird observait que 70\% des littoraux sableux à travers le monde reculaient. Le recul des côtes d'accumulation du Finistère est donc moins important (40\%). Ceci peut trouver son origine dans les nombreuses mesures mises en place depuis la fin des années 1980 en vue de réguler la pression humaine, notamment sur les espaces dunaires, et d'en diminuer ainsi les effets érosifs (GUILCHER \& HALLEGOUËT, 1991). Si l'on compare ces chiffres à ceux produits par l'Ifen (2004) pour l'ensemble des littoraux de la France métropolitaine, on s'aperçoit que la dynamique du trait de côte dans le Finistère est équivalente à la tendance nationale estimée à $48 \%$ de côtes sableuses en recul. Si l'on tient compte de ceux observés lors du programme EROCOVUL (HENAFF et al., 2006) pour l'ensemble des littoraux de la Bretagne, on observe que l'érosion du trait de côte du Finistère est supérieure à celle observée sur l'ensemble du trait de côte breton $(21,9 \%)$.

\section{Conclusion}

Pour la première fois, un bilan exhaustif de l'évolution du trait de côte à long terme est dressé pour l'ensemble dans des formes sédimentaires (sableuses et de galets) du département du Finistère. Il donne une image globale des tendances observées à l'échelle des 60 dernières années montrant ainsi que 40\% du linéaire côtier recule. Comme on pouvait s'y attendre, l'érosion affecte préférentiellement les plages ouvertes constituées de cordons dunaires et les formes fuyantes particulièrement mobiles telles que les flèches à pointe libre. Les plages adossées en position d'abri résistent mieux à l'érosion. Cette dynamique du trait de côte rejoint la tendance observée à l'échelle des littoraux français. Comme l'ont suggéré de nombreux auteurs, elle s'expliquerait en grande partie par les effets cumulés d’une crise sédimentaire et de l'élévation du niveau marin (STEPHAN, 2011 ; PASKOFF, 1998 \& 2004). 


\section{XIII ${ }^{\text {èmes }}$ Journées Nationales Génie Côtier - Génie Civil \\ Dunkerque, 2-4 juillet 2014}

\section{Remerciements :}

Ce travail a bénéficié d'une aide de l'Etat gérée par l'Agence Nationale de la Recherche au titre du programme "Investissements d'avenir" portant la référence ANR-10-LABX19.

\section{Références bibliographiques}

BIRD E.C.F. (1985). Coastline Changes. A Global Review, Wiley, Chischester, 219 p. BOAK E.H., TURNER I.L. (2005). Shoreline Definition and Detection: A Review. Journal of Coastal Research, Vol. 21(4), pp 688-703. http://dx.doi.org/10.2112/03-0071.1

CROWELL S., LEATHERMAN P., BUCKLEY M.K. (1991) Historical Shoreline Change: Error Analysis and Mapping Accuracy. Journal of Coastal Research, Vol. 7(3), pp 839-852.

CROWELL M., DOUGLAS B.C., LEATHERMAN S.P. (1997). On Forecasting Future U.S. Shoreline Positions: A Test of Algorithms. Journal of Coastal Research, Vol. 13, N 4, pp 1245-1255.

DOLAN R., HAYDEN B., HEYWOOD J. (1978). A new photogrammetric method for determining shoreline erosion. Coastal Engineering, Vol. 2, pp 21-39. http://dx.doi.org/10.1016/0378-3839(78)90003-0

DOUGLAS C., CROWELL M., LEATHERMANS P. (1998). Considerations for Shoreline Position Prediction. Journal of Coastal Research, Vol. 14(3), pp 1025-1033.

FENSTER M.S., DOLAN R., ELDER J.F. (1993). A New Method for Predicting Shoreline Positions from Historical Data. Journal of Coastal Research, Vol. 9, N 1 , pp 147-171.

GUILCHER A. (1948). Le relief de la Bretagne méridionale, de la baie de Douarnenez à la Vilaine. Henri Potier, Imprimeur - Editeur, La Roche-sur-Yon, 682 p.

GUILCHER A., HALLEGOUËT B. (1991). Coastal dunes in Brittany and their management. Journal of Coastal Research, Vol. 7(2), pp 517-533.

HALLEGOUËT B., BODERE J.C., PIRIOU N. (1986). La gestion des dunes littorales dans le Finistère. Revue Norois. $n^{\circ}$ 132. L'aménagement dans la France de l'Ouest, pp 517-535. http://dx.doi.org/10.3406/noroi.1986.4350

HENAFF A., BODERE J.C., LAGEAT Y. (2006). Erosion côtière et vulnérabilité du trait de côte en Bretagne. Rapport annuel du PRIR Bretagne. http://hal.archivesouvertes.fr/hal-00073137

MOORE L.J. (2000). Shoreline Mapping Techniques. Journal of Coastal Research, Vol. 16(1), pp 111-124.

MOORE L.J., BENUMOF B.T., GRIGGS G. (1999). Coastal Erosion Hazards in Santa Cruz and San Diego Counties, California. Journal of Coastal Research, Special Issue $n^{\circ}$ 28. Coastal Erosion Mapping and Management, pp 121-139.

PASKOFF R. (1998). La crise des plages : pénurie de sédiments. Mappemonde, Vol. 52(4), pp 11-15. 
PASKOFF R. (2004). Les littoraux sableux et dunaires : de l'abondance à la pénurie en sédiments. Bulletin de l’Association des Géographes Français, Vol. 3, pp 373-382.

PRIEST G.R. (1999). Coastal shoreline change study northern and central Lincoln County, Oregon. Journal of Coastal Research, Special Issue n²8, pp 140-157.

SHOSHANY M., DEGANI A. (1992). Shoreline Detection by Digital Image Processing of Aerial Photography. Journal of Coastal Research, Vol. 8(1), pp 29-34.

STEPHAN P. (2011). Quelques données nouvelles sur la mobilité récente (1930-2008) et le bilan sédimentaire des flèches de galets de Bretagne. Géomorphologie : relief, processus, environnement, Vol. 2, pp 205-232.

THIELER E., HIMMELSTOSS E., ZICHICHI J., ERGUL A. (2009), The Digital Shoreline Analysis System (DSAS) version 4.0, an ArcGIS extension for calculating shoreline change: U.S. Geological Survey Open-File Report 2008-1278. http://woodshole.er.usgs.gov/project-pages/dsas/version4/ 